\title{
Cerebral Tuberculoma without Clinical Pulmonary Tuberculosis Mimicry as Cerebral Abscess: (A Rare Case Report)
}

\author{
Badrul Munir ${ }^{*}$, SB Rianawati ${ }^{2}$, Catur $\mathrm{AS}^{2}$ and Dotti Inggrianita ${ }^{3}$ \\ ${ }^{1}$ Consultant Neuroinfection, Brawijaya University, Indonesia \\ ${ }^{2}$ Department of Neurology, Brawijaya University, Indonesia \\ ${ }^{3}$ Resident of Neurology Department, Brawijaya University, Indonesia
}

*Corresponding author: Badrul Munir, Consultant Neuroinfection, Faculty of Medicine Brawijaya University, Saiful Anwar General Hospital, Malang, Indonesia.

To Cite This Article: Badrul Munir. Cerebral Tuberculoma without Clinical Pulmonary Tuberculosis Mimicry as Cerebral Abscess: (A Rare Case Report). Am J Biomed Sci \& Res. 2019 - 5(4). AJBSR.MS.ID.000921. DOI: 10.34297/AJBSR.2019.05.000921.

Received: 留August 31, 2019; Published: 眥 September 26, 2019

\begin{abstract}
Cerebral tuberculoma is approximately $10-15 \%$ of intracranial tuberculosis cases and $1 \%$ of all Mycobacterium tuberculosis (MTB) cases. Mostly, Cerebral tuberculoma is always accompanied by pulmonary tuberculosis infection. However, clinical and radiological symptoms of cerebral tuberculoma has mimicry with other brain infections. Gold standard of cerebral tuberculoma is histopathological finding, but it isn't hard to do so MRI and MRI spectroscopy are used to make sure this diagnosis. In this case we will explore cerebral tuberculoma without clinical pulmonary tuberculosis has mimicry cerebral abses.

Case Presentation: 20-years-old woman, complained a progressive left side body weakness accompanied by an intermittent headache for 6 months ago. She had also complained a discharge appeared from both ears for 7 months ago. Patient's contact history of tuberculosis was doubtful. Hemiparese dextra and smelly yellowish discharge from both ears was found from the examination. Neurological examination: there is no meningeal sign, patient has left hemiparese, the discharge and sputum Gene expert test did not show any sign of MTB. Weber test showed no lateralization and Rinne was negative. Based on contrast head MRI, Multiple lesions in cerebellum, right parietal lobe, and left occipital lobe accompanied by cerebral edema was found, suggestive of cerebral abscess. However, subsequent examination using MR spectroscopy suggested a brain tuberculoma. Oral Anti tuberculosis therapy had shown satisfying result.
\end{abstract}

Conclusion: There are similarities in clinical symptoms and radiological findings between cerebral tuberculoma and cerebral abscess, it is necessary to have MR spectroscopy as a substitution if there are no histopathological examination available.

Keywords: Cerebral tuberculoma; Cerebral abscess; MR spectroscopy

\section{Introduction}

Cerebral tuberculoma is one of the rare extrapulmonary tuberculosis infection that needs serious concern, due to hematogenous spread [1,2]. Positive-smear TB patients is the main source of transmission. When a TB patient coughs or sneeze, the bacteria will spread to the air in the form of sputum droplets (droplet nuclei) $[2,3]$. Cerebral tuberculoma is approximately $10-15 \%$ of intracranial tuberculosis cases and 1\% of all Mycobacterium tuberculosis (MTB) cases [4,5]. The diagnosis of cerebral tuberculoma still requires a careful examination and has quite complicated method. Until now, the proper technique and method for diagnosing and managing cerebral tuberculoma is still questionable. The gold standard for cerebral tuberculoma is caseosa granuloma found in tissue sample histopathology along with the central necrosis surrounded by histiocytes epithelium. Radiological examination can help diagnosing the cerebral tuberculoma. The radiological imaging of cerebral tuberculoma can resemble some other diseases like cerebral abscess, cerebral toxoplasma, metastatic tumors, and other central nervous system diseases.

\section{Case Presentation}

Women, 20 years-old admitted to Saiful Anwar General Hospital Malang complaining of a progressive left side body weakness accompanied by an intermittent headache for 6 months ago. The patient also complained hearing loss in both ears gradually for 7 months ago followed by yellowish discharge in both ears. A contact 
history with TB patient is still unclear, but the patient's schoolmate had been said to have a lung disease.

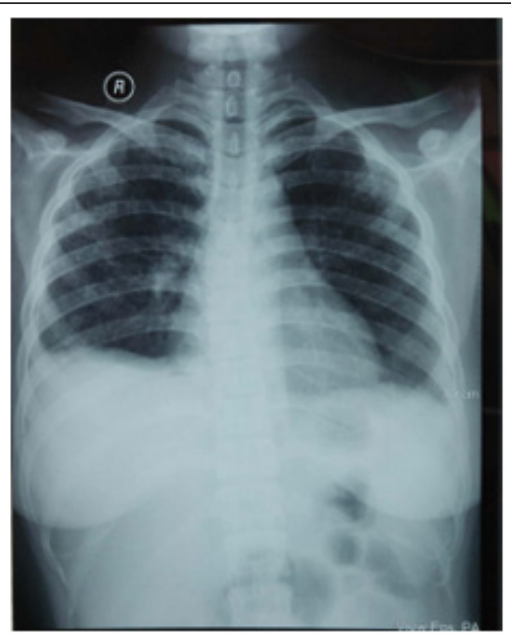

Figure 1: $\mathrm{X}$ ray examination: infiltrat millier and pleura efussion suspect infection lung TB.

Physical examination showed a good patient consciousness with GCS of 456, right hemiparese with the upper limb and lower limb power were 2 and 4 respectively, and presence of smelly yellowish discharge in both ears. Weber test showed no lateralization and Rinne was negative. The discharge culture result showed colony of Pseudomonas aeruginosa sensitive with gentamycin, ceftazidime, and meropenem, but resistance to ciprofloxacin and levofloxacin. The discharge Ziehl Neelsen test and Gene expert result showed negative result. Sputum gene expert result also showed negative result. Chest $\mathrm{X}$ ray and sputum examination gen expert didn't detect tuberculosis infection (Figure 1). Head contrast MRI showed multiple lesions in cerebellum, right parietal lobe, and left occipital lobe accompanied by cerebral edema, suggestive of cerebral abscess (Figure 2). We have given intravena antibiotik for management of cerebral abscess for 14 days but there is no clinical improvement, we performed head MR spectoscopy and suggested cerebral tuberculosis with increased lactate, NAA degradation, and increased choline/creatine ratio in lesion (Figure 3). The administration of HRZS (Isoniazid, Rifampicin, pyrazinamide, streptomycin) oral anti tuberculosis therapy according to the guidelines for intracranial TB therapy gave satisfactory results with clinical and radiological improvement.

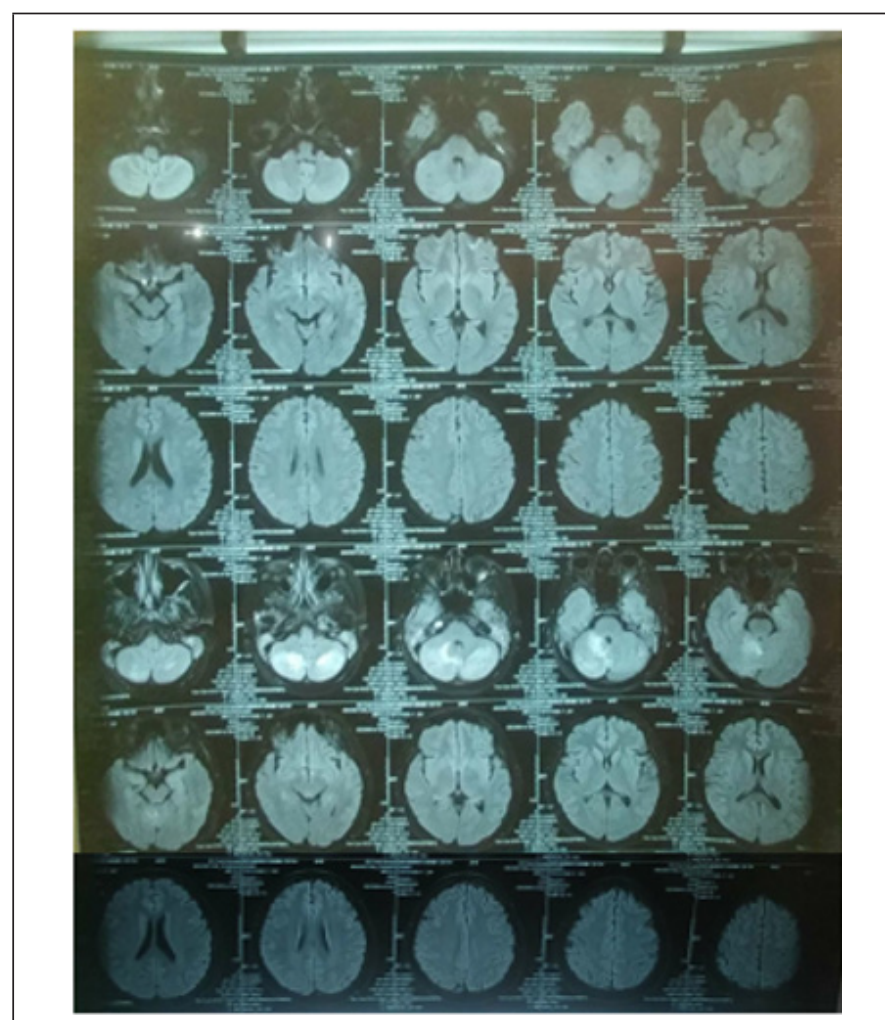

Figure 2: Head MRI: Multiple lession in right cerebellar and left parietal with edema cerebri sugesting absess cerebri.

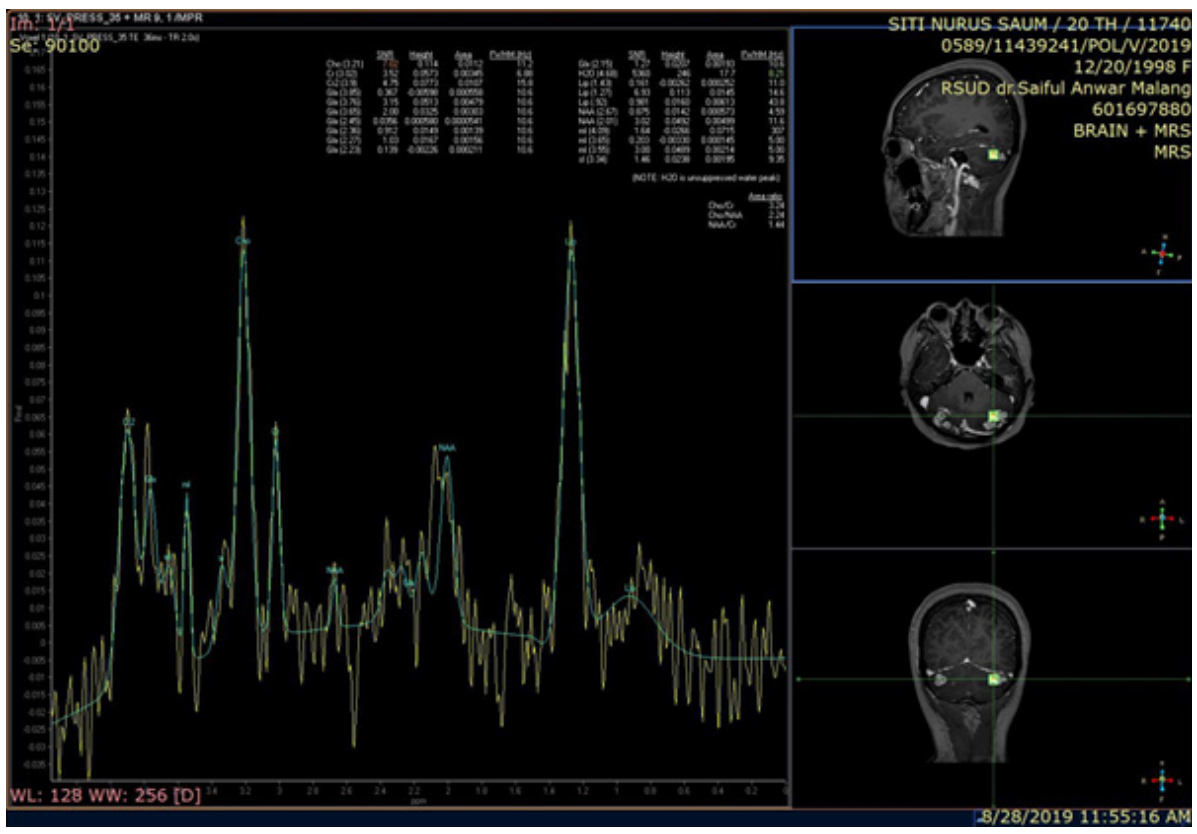

Figure 3: MR Sepctoscopy: show increased lactate, NAA degradation, and increased choline/creatine ratio in lesion. 


\section{Discussion}

Since 2003, there has been 9,5 million of new tuberculosis cases around the world. According to CDC, in 2005, there were approximately $6.3 \%$ of extrapulmonary tuberculosis cases involving the central nervous system (1.3\% of all tuberculosis cases) [6]. Intracranial tuberculosis consists of TB meningitis, TB encephalitis, cerebral tuberculoma, and intracranial tuberculosis vasculitis. TB meningitis is the most intracranial tuberculosis type occurred with total of $40 \%$ while tuberculoma is the second most [5].

Cerebral tuberculoma has ambiguous clinical presentations. The clinical presentations appeared usually are caused by space occupying lesion. The most symptoms presented in this disease are seizure (60\%-100\%), increased intracranial pressure signs (56\%$93 \%$ ), and focal neurological deficit (33\%-68\%) [6]. Radiological examination that can be applied to diagnose the tuberculoma is contrast head MRI and MRI spectroscopy [6,7].

When ring enhancement lesion is found in CT scan or MRI, MR spectroscopy has high diagnostic value for diagnosing tuberculoma. MR spectroscopy will show high lipid concentration, reduced NAA and creatinine concentration, and creatinine/choline ration $>1$. The high lipid concentration in MR Spectroscopy along with the ring enhancement lesion are specific for tuberculoma and are not seen in any differential diagnosis for tuberculoma. MR Spectroscopy can also distinguish tuberculoma and bacterial tuberculosis abscess. In TB abscess, MR Spectroscopy will show a high level of lactate and significant acetate presentation [6].

Abscess is one of the differential diagnosis for tuberculoma. The clinical sign and symptoms depend on size and site of the abscess. In general, the triad sign and symptoms for abscess are headache, fever, and neurological deficit. These signs often manifest in $2-3$ weeks gradually and progressive $[5,8]$.

In our case, we found sub-acute to chronic progressive left hemipharesis with accompanied by cephalgia, there are no anamnesis matched with typical signs of certain intracranial infection. However, with the presence of discharge in both ears can narrow down the diagnosis to abscess with the suspicion of percontinuitatum spread, tuberculoma with tuberculosis otitis, or lung tuberculosis. Therefore, physical and laboratory examination are needed to help diagnosis the patient [9]. Complete blood count (CBC), blood sedimentation rate (BSR), blood glucose, electrolyte serum, ureum/ creatinine, SGOT/SGPT, Albumin, TB ICR, discharge and sputum gene expert, and discharge examination using Ziehl Neelsen are the laboratory examination required for diagnosis. The increased BSR showed positive result with $70 \mathrm{~mm} /$ hour. The discharge culture result showed colony of Pseudomonas aeruginosa sensitive with gentamycin, ceftazidime, and meropenem, but resistance to ciprofloxacin and levofloxacin [10]. The discharge Ziehl Neelsen test and Gene expert result showed negative result. Sputum gene expert result also showed negative result. Based on these results, it can be concluded that the patient had chronic suppurative otitis media caused by Pseudomonas aeruginosa and eliminated the possibility of tuberculosis otitis which was suspected as the source of abscess infection. Lung tuberculosis as the major companion of extra pulmonary tuberculosis is not found in this patient.

MR Spectroscopy result showed isointenseT1W1 intraaxial lesion, hyper intense T2W1/FLAIR at non restricted central DWI, some with SWI blooming, Multiple in the right and left cerebellum hemisphere and in the right parietal lobe subcortex which worsen the rim after contrast administration. The largest diameter was $8.2 \mathrm{~mm}$ in right cerebellum hemisphere. There was lipid degradation, increased lactate, NAA degradation, and increased choline/ creatine ratio in lesion, compared to the perilesion tissue. Furthermore, multiple intraaxial lesions were found in bilateral cerebellum hemisphere and right sub cortex suspected with tuberculoma (improved), bilateral chronic mastoiditis (fixed) (Figure 2).

In normal brain, MR Spectroscopy will show the predominant $\mathrm{N}$-acetylaspartate (NAA), choline, creatine, and myo-inositol with highest spectrum on NAA. NAA is neuronal marker for healthy tissue, choline shows energy deviation and is cellular marker [6].

\section{Conclusion}

Cerebral tuberculoma is quite rare and the gold standard for diagnosis of tuberculoma is histopathological examination, but it is rare to performed, so head MR Spectroscopy is considered for distinguish the diagnosis possibilities to make sure cerebral tuberculoma has mimicry others brain infection, before give administered oral anti tuberculosis therapy.

\section{References}

1. Monteiro v Regina, Carneiro José Carlos, Costa Claúdia, Duarte Raquel (2013) Cerebral tuberculomas-A clinical challenge. Respiratory Medicine Case Reports 9: 34-37.

2. B Munir, SB Rianawati, H Sutanto, UA Setyawan (2017) Mortality of central nervous system infection adult patients in indonesia (Descriptive study in a tertier referral hospital). Journal of the Neurological Sciences 381: 414 .

3. B Munir, D Candradikusuma (2015) Manifestation of HIV AIDS in Case Neurology at Saiful Anwar Hospital 2013-2014. MNJ 1(1): 7-11.

4. (2011) Kementerian Kesehatan Republik Indonesia Direktorat Jenderal Pengendalian Penyakit Dan Penyehatan Lingkungan [Minister of Health General Directorate of Disease Control and Environmental Health] (2 ${ }^{\text {nd }}$ edn), Pedoman Nasional Pengendalian Tuberkulosis, Kementerian Kesehatan Republik Indonesia [National Guidelines for Tuberculosis Control. Ministry of Health of the Republic of Indonesia], Jakarta, Indonesia.

5. Rock BR, Olin Michael, Baker CA, Molitor TW, Petterson PK (2008) Central Nervous system Tuberculosis: Pathogenesis and Clinical Aspects. Clinical Microbiology Review, Apr 2008. Minnesotta pp. 243-261.

6. (2016) Pusat Data dan Informasi Kementerian Kesehatan RI [Data and Information Center of Ministry of Health Republic of Indonesia], Jakarta, Indonesia.

7. Sharma SK, Mohan A (2004) Extrapulmonary tuberculosis. Indian J Med Res pp. 316-353.

8. Mukherjee Subhasis, Das Runa, Begum Shabana (2015) Tuberculoma of the brain - A diagnostic dilemma: Magnetic resonance spectroscopy a new ray of hope. The Journal of Association of Chest Physicians 3(1): 3-8. 
9. Khatri Garvit D, Krishnan Venkatram, Antil Neha, Saigal Gaurav (2018) Magnetic resonance imaging spectrum of intracranial tubercular lesions: one disease, many faces. Pol J Radiol 83: e524-e535.
10. Riddell J, Shuman EK (2012) Epidemiology of Central Nervous System Infection. Neuroimag Clin N Am 22(4): 543-556. 